\title{
Electromagnetic field intensity distribution along focal region of a metallic circular reflector covered with a plasma layer
}

\author{
A. Ghaffar \\ aghaffar@ksu.edu.sa \\ M. A. S. Alkanhal \\ majeed@ksu.edu.sa
}

\author{
Department of Electrical Engineering, King Saud University, Saudi Arabia \\ Department of Physics, University of Agriculture, Faisalabad, Pakistan \\ Department of Electrical Engineering, King Saud University, Saudi Arabia
}

Theoretical analyses has been carried out to study the deviation of the electromagnetic field intensity distribution in the focal region of a long metallic circular reflector that contains a uniform cold collisional plasma layer on its surface. The electromagnetic field intensity expressions along the focal region have been obtained using Maslov's method. Maslov's method is systematic procedure, which combines the simplicity of ray optics and the generality of transform methods. The derived analytical field expressions in the focal region have been solved numerically. The reflected and transmitted field intensity distributions from the plasma layer along the focal point were examined. The effects of some physical parameters such as the plasma frequency, the thickness of plasma layer and the effective collision frequency on the transmitted field intensity distribution along the focal region are studied. The results are found to be in a good agreement with results obtained using Kirchhoff's approximation.

[DOI: http://dx.doi.org/10.2971/jeos.2015.15014]

Keywords: Circular reflector, field intensity distribution, focal point, Maslov's method, plasma layer

\section{INTRODUCTION}

In recent years the study of analysis of electromagnetic waves in the focal region by plasma as absorbers or reflector $[1,2]$ received substantial attention from researchers. Analysis of such fields is important in the current arena of advanced technologies for microwave, millimeter-wave, and optical device applications. The analysis of focal region filed is useful for optical spectroscopy, medical treatment and hyperthermia. The image field may be also useful to generate images of the human body with the help of radio frequency technologies. Such type of analysis is very significant to find out the suitable parameters of the plasma which affect the reflection, absorption, and transmission of the electromagnetic energy [3]. When cylindrical and spherical metallic structures, as reflector antennas for example, enter into the Earth's atmosphere with high velocities, plasma layer forms on their surfaces [4].Thus, usually antennas of space vehicles are in the surface contact with the plasma layer and this layer affects the radiation characteristics of such antennas. Also, the existence of the plasma layer on a metallic target changes the reflected wave energy, which is an important issue especially in the study of the interaction of intense electromagnetic waves with a metallic surface.

Many types of reflectors have been investigated in the open literature for efficiently focusing electromagnetic waves for different applications. Among these reflector shapes are elliptical reflectors, cylindrical reflectors, hyperbolic reflectors, and parabolic reflectors $[5,6]$. These reflectors are used as reflective devices used to collect or project the power of the electromagnetic waves. The opposite is also true; an electromagnetic wave source placed at the foci of a reflector produces a parallel beam of electromagnetic waves. The circular reflector is commonly used for microwave, millimeter-wave, and optical device applications.

The geometrical optics (GO) approximation is a well-known technique used to analyze high frequency electromagnetic fields. However, in many problems, such as describing fields in the vicinity of focal region, geometrical optics (GO) does not provide satisfactory results [7]. To overcome the defect of GO, Maslov's method is used. Maslov's method is a combination of asymptotic ray theory (ART) and Fourier transform method [8]-[11]. According to Maslov's method, the ray is expressed in terms of six coordinates i.e., components of wave vectors and space coordinates. The conventional ray expression may be considered as its projection in the space coordinates. At the singular point of the ray-expression in space coordinates, the six-component expression is employed. The expression in the hybrid coordinates may be transformed into that of space coordinates through Fourier transform. This technique has been used to study high frequency fields in the focal region successfully by many authors [12]-[15]. Huygens Kirchhoff's integral is another alternative technique. It is based on a short Fresnel approximation. Generally, Huygens Kirchhoff's integral and Maslov's method are of comparable accuracy. However, Maslov's method has a distinct advantage for specific problems, such as the transmission through a focusing system with multiple interfaces [16].

In this paper, we consider a long metallic circular reflector cov- 
ered with a cold collision plasma layer on its surface. It is presented that reflected field intensity from this circular reflector and by assuming circular plasma layer on the surface of the reflector, a deviation of field intensity distribution occur along the focal point.The effects of some physical parameters such as the plasma frequency, the thickness of the plasma layer and the effective collision frequency on the transmitted field intensity distribution along the focal points have been studied. The results of the presented formulations have been compared to Kirchhoff's integral, which confirms the accuracy of the presented analysis. The time-harmonic $(i \omega t)$ dependence is adopted and suppressed in what follows.

\section{FORMULATIONS}

Consider a circular reflector made of perfect metal in the presence of a plasma layer as shown in Figure 1. It is assumed that an electromagnetic plane wave is incident normally on the surface of the reflector parallel to its symmetry axis. The equation of the metallic circular reflector is given by

$$
\zeta=\sqrt{a^{2}-\xi^{2}}
$$

where $a$ is the radius of the metallic circular reflector. Then, the equation of the circular plasma layer placed on the metallic circular reflector is defined as follows:

$$
\zeta=\sqrt{p^{2}-\xi^{2}}
$$

where $p=\sqrt{a^{2}-d^{2}}$ is the radius of the circular plasma layer, $a$ is the radius of the circular metallic circular reflector and $d$ is the thickness of the plasma layer. Coordinates of a point on the circular surface of the plasma layer $P\left(\xi_{0}, \zeta_{0}\right)$ in terms of a point on the metallic circular reflector $Q(\xi, \zeta)$ are defined as [4]

$$
\begin{aligned}
& \xi_{0}=\xi-d \sin \alpha \\
& \zeta_{0}=\zeta-d \cos \alpha
\end{aligned}
$$

Now, we consider a monochromatic electromagnetic wave polarized in the y-axis normally incident on the circular plasma layer, parallel to its symmetry axis as

$$
E_{0 i}=E_{i} \hat{e}_{y} \exp \left(-j k_{i} z\right)
$$

The refractive index of the collisional plasma is defined as

$$
\mathrm{n}=\sqrt{1-\frac{j \omega_{p}^{2}}{(v+j \omega) \omega}}
$$

where $\omega_{p}$ is the plasma frequency and $\mathrm{V}$ is the effective collisional frequency. The wave vector of the reflected wave and the transmitted wave can be worked out using Snell's laws whose mathematical expression is described in the following form

$$
\begin{aligned}
& \mathbf{p}^{\mathbf{r}}=\mathbf{p}^{\mathbf{i}}-2\left(\mathbf{p}^{\mathbf{i}} \cdot \mathbf{N}\right) \mathbf{N} \\
& \mathbf{q}^{\mathbf{r}}=\mathbf{p}^{\mathbf{i}}+\sqrt{n^{2}-1+\left(\mathbf{p}^{\mathbf{i}} \cdot \mathbf{N}\right)^{2}} \mathbf{N}-\left(\mathbf{p}^{\mathbf{i}} \cdot \mathbf{N}\right) \mathbf{N}
\end{aligned}
$$

where $\mathrm{p}^{\mathrm{i}}$ is the wave vector of the incident wave, $n$ is refractive index of the plasma medium and $\mathbf{N}$ is unit vector normal to

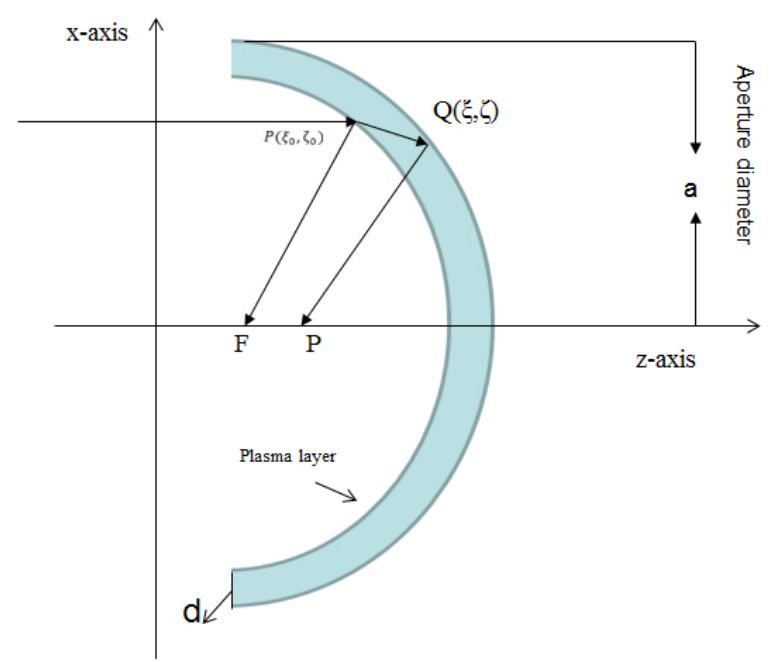

FIG. 1 Circular reflector made of perfect metal with a layer of plasma on its surface.

the surface of the plasma coated circular reflector and it can be written as

$$
\mathbf{N}=\hat{e}_{z} \cos \alpha+\hat{e}_{x} \sin \alpha
$$

By applying Snell's law of reflection and refraction, the wave vector $\mathbf{p}_{1}$ of the reflected wave from the plasma layer, the wave vector $\mathbf{q}_{\mathbf{1}}$ of the refracted wave into the plasma layer, the wave vector $\mathbf{p}_{2}$ of the reflected wave from the metallic reflector, and the wave vector $\mathbf{q}_{2}$ of the refracted wave out of the plasma circular layer into free space are given by [11]

$$
\begin{aligned}
\mathbf{p}_{1}= & -\hat{e}_{x} \sin 2 \alpha-\hat{e}_{z} \cos 2 \alpha \\
\mathbf{q}_{1}= & \sin \alpha\left(-\cos \alpha+\sqrt{n^{2}-\sin ^{2} \alpha}\right) \hat{e}_{x} \\
& -\left(\cos \alpha\left(-\cos \alpha+\sqrt{n^{2}-\sin ^{2} \alpha}\right)+1\right) \hat{e}_{z} \\
\mathbf{p}_{2}= & -\hat{e}_{x}\left(K_{1} \sin \alpha+\cos \alpha \sin \alpha\right) \\
& -\hat{e}_{z}\left(K_{1} \cos \alpha-\sin \alpha \sin \alpha\right) \\
\mathbf{q}_{2}= & -\hat{e}_{x}\left(K_{2} \sin \alpha+n \cos \alpha \sin \alpha\right) \\
& -\hat{e}_{z}\left(K_{2} \cos \alpha-n \sin \alpha \sin \alpha\right)
\end{aligned}
$$

where

$$
\begin{aligned}
& K_{1}=\sqrt{\left(-1+2 n^{2}+\cos 2 \alpha\right) / 2} \\
& K_{2}=\sqrt{(2+n(-1+2(-1+n) n)+n \cos 2 \alpha) / 2} .
\end{aligned}
$$

Using the components of the reflected wave and the transmitted wave respectively, the solutions of the Hamilton's equations are

$$
\begin{array}{ll}
x=\xi+p_{1 x} \tau_{1} & z=\zeta_{0}+p_{1 z} \tau_{1} \\
x=\xi+q_{1 x} \tau_{2} & z=\zeta_{0}+q_{1 z} \tau_{2}
\end{array}
$$

The Jacobian associated with the wave reflected by the circular plasma layer is obtained as

$$
\mathrm{J}_{1}\left(\tau_{1}\right)=\frac{\mathrm{D}_{1}\left(\tau_{1}\right)}{\mathrm{D}_{1}(0)}=\tau_{1}-\frac{\cos \alpha}{2} p
$$

The Jacobian associated with the wave refracted by the plasma 
circular layer is obtained as

$$
\begin{aligned}
\mathrm{J}_{2}\left(\tau_{2}\right)= & \frac{\mathrm{D}_{2}\left(\tau_{2}\right)}{\mathrm{D}_{2}(0)} \\
= & \frac{1}{\sqrt{2} \alpha K_{2}^{2}}(\sqrt{2} \alpha(2+n(-1+2(-1+n) n)) \\
& +K_{2}\left(-2+n+n^{2}-2 n^{3}\right) \tau_{2} \\
& -2 \sqrt{2} n\left(1+(-1+n) n^{2}\right) \tau_{2} \cos \alpha \\
& \left.+n\left(\sqrt{2} \alpha+K_{2}(-1+n) \tau_{2}\right) \cos 2 \alpha\right)
\end{aligned}
$$

The field expressions for the reflected and transmitted rays out of the plasma layer in geometric optics are obtained as

$$
\begin{aligned}
& E_{r}(x, z)=E_{i}\left[J_{1}\left(\tau_{1}\right)\right]^{-1 / 2} \exp \left[-j k\left(\Psi_{0}+\tau_{1}\right)\right] \\
& E_{t}(x, z)=E_{i}\left[J_{2}\left(\tau_{2}\right)\right]^{-1 / 2} \exp \left[-j k\left(\Psi_{0}+\tau_{2}+t\right)\right]
\end{aligned}
$$

where $\Psi_{0}=p \cos a, \tau_{1}$ and $\tau_{2}$ are parameter along the ray reflected from the circular plasma layer and transmitted ray out of the circular plasma layer to focal point respectively and $t=\sqrt{\left(\xi_{0}-\xi\right)^{2}+\left(\zeta_{0}-\zeta\right)^{2}}$ is the distance between $P\left(\xi_{0}, \zeta_{0}\right)$ and $Q(\xi, \zeta)$. It is observed that the GO reflected and transmitted fields become infinity at the caustic or focal points as expected when $J(\tau)=0$. We can derive the expression which is valid at the focal point using the Maslov's method. The exact location of the focal or caustic point can be obtained at $J(\tau)=0$. According to Maslov's method field expressions valid in the focal region can be written as below [12]-[15].

$$
\begin{aligned}
\mathrm{E}^{r}(r)= & \sqrt{\frac{\mathrm{k}}{\mathrm{j} 2 \pi}} \int_{-\infty}^{\infty}\left[\frac{D(\tau)}{D(0)} \frac{\partial\left(p_{z}\right)}{\partial(z)}\right]^{-\frac{1}{2}} \\
& \exp \left\{-\mathrm{jk}\left[S_{0}+\tau-z\left(p_{x}, z\right) p_{z}+p_{z} z\right]\right\} d p_{z}
\end{aligned}
$$

Integrands of the integrals of the reflected and transmitted fields from the plasma layer are obtained, respectively, as

$$
\begin{aligned}
\mathrm{I}_{1}= & \frac{D\left(\tau_{1}\right.}{D(0)} \frac{\partial\left(p_{1 x}\right)}{\partial(x)}=\frac{2 \sin ^{2} 2 \alpha}{p \cos \alpha} \\
\mathrm{I}_{2}= & \frac{D\left(\tau_{2}\right.}{D(0)} \frac{\partial\left(p_{2 x}\right)}{\partial(x)}=\frac{1}{2 p K_{2}}\left(2 n ^ { 2 } \operatorname { c o s } \alpha \left(K_{2}+\cos \alpha \sin ^{2} \alpha\right.\right. \\
& +K_{2} \sin \alpha\left(4 K_{2} n \sin \alpha+n(1+n) \sin 2 \alpha\right. \\
& \left.\left.+2 K_{2}\left(K_{2}+n \cos \alpha\right) \tan \alpha\right)\right)
\end{aligned}
$$

The phase functions of the integrals for the reflected and transmitted fields from the plasma layer are obtained, respectively, as

$$
\begin{aligned}
S_{1}= & S_{0}+\tau_{1}-z\left(x, p_{z}\right) p_{z}+p_{x} x \\
S_{1}= & p \cos \alpha+\sqrt{(x-p \sin \alpha)^{2}+(z-p \cos \alpha)^{2}} \\
S_{2}= & S_{0}+\tau_{2}+t-z\left(x, p_{z}\right) p_{z}+p_{x} x \\
S_{2}= & p \cos \alpha+p \mathrm{~K}_{2}-\mathrm{K}_{2} r \cos \alpha+\alpha \cos \alpha \\
& +n r \sin (\alpha+\theta) \sin \alpha+t
\end{aligned}
$$

Using Eqs. (19) and (21) into (18) and changing the variable $p_{1} z$ into $a$, given in Eq. (10), yields the final finite reflected field expression valid along the focal point a

$$
\frac{E(x, z)}{E_{i}}=\sqrt{\frac{k \alpha}{\pi}} \int_{-l / 2}^{l / 2} \frac{1-n}{1+n} \sqrt{\cos \alpha} \exp \left[-j k S_{1}\right] d \alpha
$$

Again using Eqs. (19) and (21) into (17) and changing the variable $p_{2} z$ into $\alpha$, given in (11), yields the final finite transmitted field expression valid along the focal point as

$$
\begin{aligned}
\frac{E(x, z)}{E_{i}}= & \sqrt{\frac{k \alpha}{\pi}} \int_{-l / 2}^{l / 2} \frac{2 n \cos \alpha}{n \cos \alpha+\sqrt{1-n^{2} \sin ^{2} \alpha}} \\
& \times \sqrt{\frac{1}{q_{2 x}} \frac{d q_{2 z}}{d \alpha} \cos \alpha \times \exp \left[-j k\left(p \cos \alpha+p K_{2}\right.\right.} \\
& \left.-K_{2} r \cos \alpha+\alpha \cos \alpha+n r \sin (\alpha+\theta) \sin \alpha+t\right] d \alpha
\end{aligned}
$$

\section{HUYGENS-KIRCHHOFF'S INTEGRAL}

It is interesting to compare the above expression with the following formula implied by Huygens- Kirchhoff's Integral

$$
\begin{aligned}
& E(x, z) \\
& =\sqrt{\frac{k}{j 2 \pi}} \int_{-\infty}^{\infty} \frac{e^{-j R}}{R} E_{0}\left(\xi_{0}, \zeta_{0}\right) \exp \left(-j k S_{0}\right) d \xi_{0}
\end{aligned}
$$

Where $R=q_{1 x}\left(x-\xi_{0}\right)+q_{1 z}\left(z-\zeta_{0}\right)=\sqrt{\left(x-\xi_{0}\right)^{2}+p^{2}}$ and $E_{0}\left(\xi_{0}, \zeta_{0}\right)=J^{-\frac{1}{2}}$.

Therefore

$$
E(x, z)=\sqrt{\frac{k}{j 2 \pi}} \int_{-l / 2}^{l / 2} \frac{J^{-\frac{1}{2}}}{R} \times \exp \left[-j k\left(S_{0}+R\right) d \xi_{0}\right.
$$

\section{RESULTS AND DISCUSSION}

Results of reflection and transmission of a normal incident perpendicular polarized electromagnetic plane wave by a circular reflector covered with a plasma layer are presented in this section. The frequency of the incident electromagnetic wave is taken in the microwave region. Numerical results obtained from Maslov's method are compared with the results of the Huygens-Kirchhoff integral to check the correctness of the analytical calculations. Figure 2 shows the comparison of the numerical result using Maslov's method (solid line) and using Kirchhoff's approximation (dashed line) which show that the agreement is very good.

Figures 3(a)-(b) shows the comparison of the field intensity distribution of the reflected (solid line) and the transmitted (dashed line) field intensities from the circular reflector in presence of the plasma layer along the focal points with respect to $k x$ and $k z$, respectively, at $k a=5$, $\omega_{p}=1.7829 \times 10^{8} \mathrm{~Hz}, V=1 \times 10^{5} \mathrm{~Hz}$, and $\omega=3.14 \times 10^{8} \mathrm{~Hz}$. From this figure, it is observe that the maximum field intensity of the reflected field is higher as compared to the maximum field intensity of the transmitted field. It is also observed that the location of the maxima field intensity of both the transmitted and the reflected fields do not change along the $\mathrm{x}$-axis but the location of the maximum field intensity of the transmitted field intensity slightly shifts toward the reflector.

Figures 4-6 show, respectively, the effects of the plasma layer thickness, the plasma frequency, and the effective collisional 


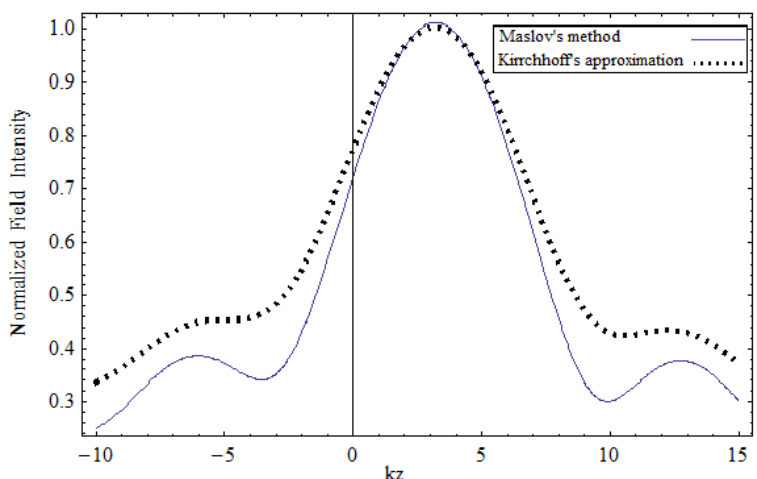

FIG. 2 Normalized field intensity by Maslov's method (solid line) and by Kirchhoff's integral (dashed line).
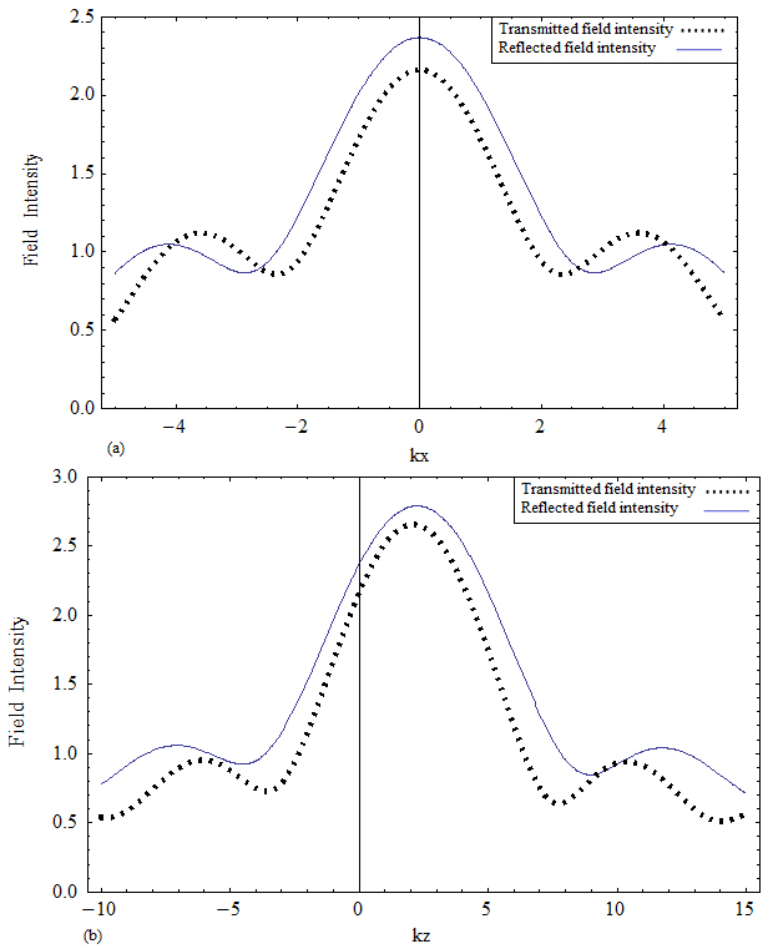

FIG. 3 Normalized field intensity distribution of the transmitted field (dashed line) and the reflected field (solid line) using Maslov's method (a) versus $k x$ (b) versus $k z$

frequency on the distribution of the transmitted field intensity from the plasma layer versus $k x$ and $k z$ respectively. Figures $4(\mathrm{a})$-(b) shows the transmitted field intensity versus $k x$ and $k z$, respectively, for different values of the plasma layer thickness $k d$. These figures have been plotted for a constant radius of the metallic circular reflector $k a=5$, wave frequency $\omega=3.14 \times 10^{8} \mathrm{~Hz}$, plasma frequency $\omega_{p}=1.7829 \times 10^{8} \mathrm{~Hz}$, and effective collisional frequency $V=1 \times 10^{5} \mathrm{~Hz}$. It is clearly observed that if thickness of the plasma layer increases the maximum value of the transmitted field intensity increases along $\mathrm{x}$-axis and slightly decreases along the z-axis. The location of maximum field intensity shifts away from the curved surface along the z-axis.

Figures 6(a)-(b) shows the changes of the transmitted field intensity versus $k x$ and $k z$, respectively, for different value of the effective collision frequency $V$ for a constant radius of the metallic circular reflector $k a=5$, wave frequency
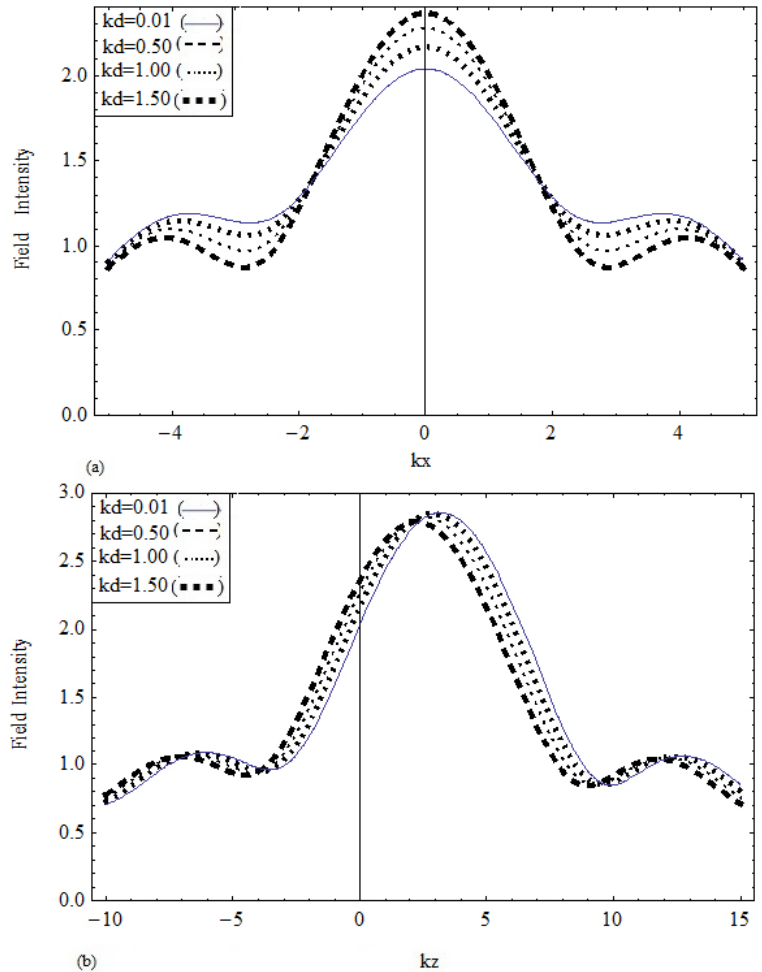

FIG. 4 Field intensity distribution of the transmitted field from the plasma layer for different values of the plasma layer thickness (a) versus $k x$ (b) versus $k z$.
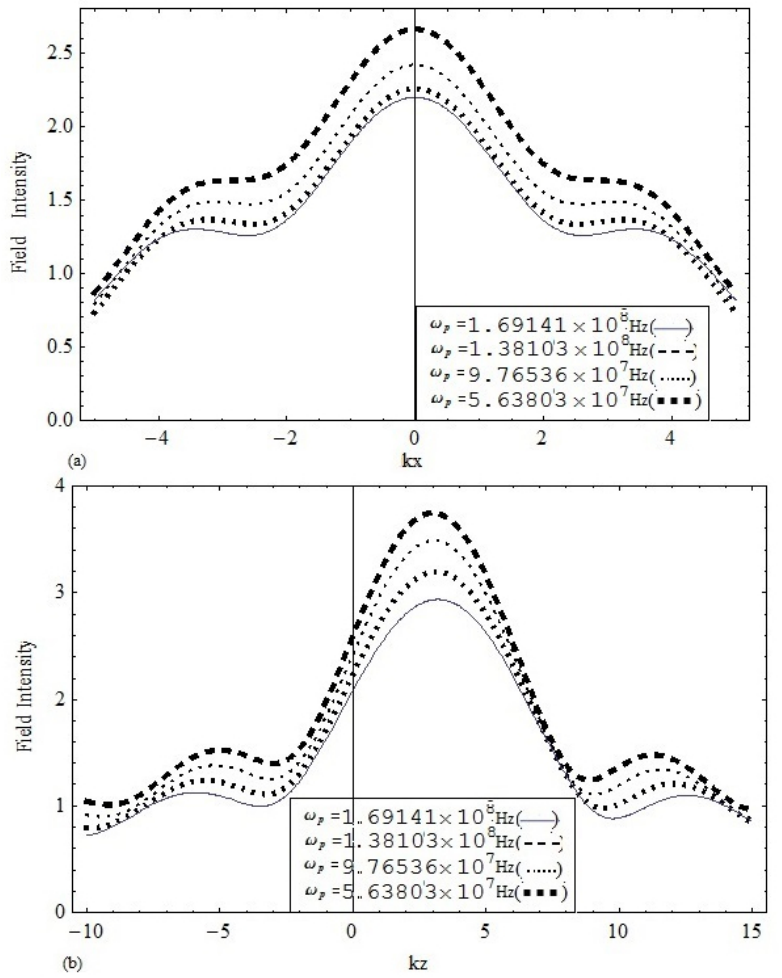

FIG. 5 Field intensity distribution of the transmitted field from the plasma layer for different values of the plasma frequency (a) versus $k x$ (b) versus $k z$.

$\omega=3.14 \times 10^{8} \mathrm{~Hz}$, plasma layer thickness $k d=5$, and plasma frequency $\omega_{p}=1.7829 \times 10^{8} \mathrm{~Hz}$. It is demonstrated that the effective collision frequency $V$ of the plasma layer has no effect on the transmitted field intensity. It is due to that the effective collision frequency $V$ is much smaller than the wave frequency of the incident wave. 

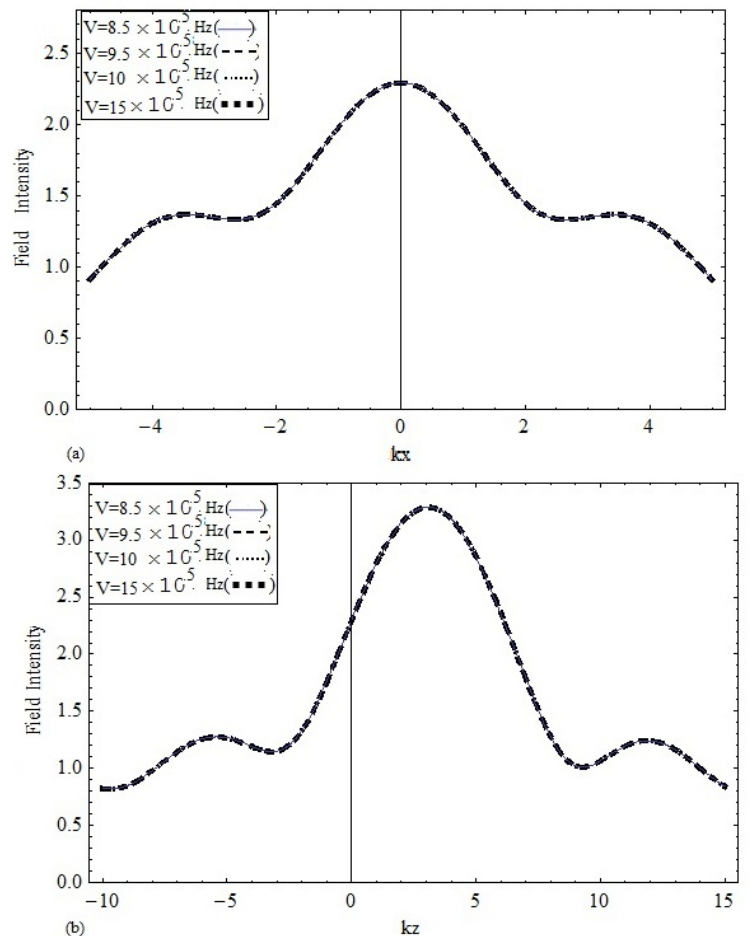

FIG. 6 Field intensity distribution of the transmitted field from the plasma layer for different values of the collision frequency of the plasma layer (a) versus $k x$ (b) versus $k z$.

\section{CONCLUSIONS}

In this paper, the nonconformity in the field intensity distribution in the focal region of a long metallic circular reflector caused by a plasma layer covering the reflector surface has been studied. Using Maslov's method, the reflected and the transmitted fields out of the plasma layer were obtained. The results were found in to be in a good agreement with results obtained using Kirchhoff's integral approximation. The effects of the plasma layer thickness, the plasma frequency, and the effective collisional frequency on the energy distribution were presented. It was shown that the transmitted field intensity has a lower value as compare to the reflected field intensity from the plasma layer. Furthermore, it is observed that when the plasma layer thickness increases, the maximum value of the transmitted field intensity decreases with respect to the $\mathrm{z}$ axis and increases with respect to the x-axis, whereas if the plasma frequency increases, the maximum value of the transmitted intensity decreases at both axes. In the studied cases, it is noted that if the thickness of the layer increases, then the maximum peak shifts away from the curved interface and that the effective collisional frequency has no effect on the transmitted field intensity. These observations should be useful in the study and design of modern optical devices.

\section{ACKNOWLEDGEMENT}

The authors would like to extend their sincere appreciation to the Deanship of Scientific Research (DSR) at King Saud University for its funding of this research through the Research Group Project no RG-1436-001.

\section{References}

[1] D. R. Nicholson and D. R. Nicholson, Introduction to plasma theory (Cambridge University Press, Cambridge, 1983).

[2] V. Smilyanskii, "Propagation of an electromagnetic wave across a magnetic field in a parabolic plasma layer," J. Appl. Mech. Tech. Phy. 12, 366-371 (1971).

[3] A. Niknam, M. Khajehmirzaei, B. Davoudi-Rahaghi, Z. Rahmani, B. Jazi, and A. Abdoli-Arani, "Electromagnetic modeling of the energy distribution of a metallic cylindrical parabolic reflector covered with a magnetized plasma layer," Phys. Plasmas 21, 073107 (2014).

[4] B. Jazi, B. Davoudi-Rahaghi, M. R. Khajehmirzaei, and A. R. Niknam, "Energy Distribution Along the Focal Axis of a Metallic Cylindrical Parabolic Reflector Covered With a Plasma Layer," IEEE T. Plasma Sci. 42, 286-292 (2014).

[5] M. Laroussi, and J. Reece Roth, "Numerical calculation of the reflection, absorption, and transmission of microwaves by a nonuniform plasma slab," IEEE T. Plasma Sci. 21, 366-372 (1993).

[6] W. M. Manheimer, "Plasma reflectors for electronic beam steering in radar systems," IEEE T. Plasma Sci. 19, 1228-1234 (1991).

[7] C. Thomson, and C. Chapman, "An introduction to Maslov's asymptotic method," Geophys. J. Int. 83, 143-168 (1985).

[8] R. W. Ziolkowski, and G. A. Deschamps, "Asymptotic evaluation of high-frequency fields near a caustic: An introduction to Maslov's method," Radio Sci. 19, 1001-1025 (1984).

[9] K. Hongo, and Y. Ji, "High-frequency expression for the field in the caustic region of a cylindrical reflector using Maslov's method," Radio Sci. 22, 357-366 (1987).

[10] K. Hongo, Y. Ji, and E. Nakajima, "High-frequency expression for the field in the caustic region of a reflector using Maslov's method," Radio Sci. 21, $911-919$ (1986).

[11] K. Hongo, and H. Kobayashi, "Radiation characteristics of a planoconvex lens antenna," Radio Sci. 31, 1025-1035 (1996).

[12] A. Chaffar, M. Shoaib, N. Mehmood, M. Naz, M. Azam, and Q. Naqvi, "Electromagnetic field diffraction of a paraboloidal mirror under oblique incidence," Int. J. Appl. Electrom. 41, 479-494 (2013).

[13] A. Ghaffar, M. Sharif, Q. Naqvi, M. Alkanhal, F. Khalid, and S. Shukurullah, "Focusing of Electromagnetic Wave from Quartic Inhomogeneous Chiro-Slab," Appl. Comput. Electrom. 29, 478-485 (2014).

[14] A. Ghaffar, M. Arif, Q. A. Naqvi, and M. A. Alkanhal, "Radiation properties of a uniaxial chiral quadratic inhomogeneous slab under oblique incidence," Optik-International Journal for Light and Electron Optics 125, 1589-1597 (2014).

[15] A. Chaffar, M. H. Shahzad, M. Y. Naz, S. Ahmed, M. Sharif, Q. A. Naqvi, and A. A. Syed, "Analysis of electromagnetic field transmitted by a uniaxial chiral lens," J. Electromagnetic Wave. 26, 1007-1017 (2012).

[16] W. Ali, F. Ahmad, A. A. Syed and Q. A. Naqvi, "Effects of negative permittivity and/or Permeability on reflection and transmission from planar, circular cylindrical, and fractal chiral-chiral interfaces," J. Electromagnetic Wave. 29, 1006375 (2015). 